\title{
RESTRICTIONS OF SMOOTH FUNCTIONS TO A CLOSED SUBSET
}

\author{
SHUZO IZUMI
}

We first provide an approach to the recent conjecture of Bierstone-MilmanPawłucki on Whitney's old problem on $C^{d}$ extendability of functions defined on a closed subset of a Euclidean space, using the higher order paratangent bundle they introduced. For example, the conjecture is affirmative for classical fractal sets. Next, we give a sharpened form of Spallek's theorem on controllability of flatness by the values on a closed set. The multi-dimensional Vandermonde matrix plays an important role in both cases.

\section{INTRODUCTION}

There remain very simple unsolved problems in a close neighbourhood of elementary calculus. They are concerned with the values of smooth functions on a subset as follows. Let $X$ be a closed subset of a domain $\Omega$ of a Euclidean space $\mathbb{R}^{n}$.

(1) Extension problem: Find the condition for a function on $X$ to be extendable to a $C^{d}$ function on $\Omega$.

(2) Flatness problem: If $X$ is thick enough around $\xi$, the Taylor expansion of a differentiable function $f$ defined in a neighbourhood of $\xi$ is determined up to some order. Find a geometric expression of this thickness.

Whitney $\mathrm{W}_{2}$ posed the extension problem and gave a necessary and sufficient condition in the case $n=1$. Glaeser $\mathrm{G}_{1}$ solved this problem in the case $d=1$ introducing the linearized paratangent bundle. (Originally, the spelling is "paratingent" in French. cf. [B] and $\mathrm{G}_{1}$.) Recently, Bierstone, Milman and Pawłucki $\mathrm{BMP}_{2}$ introduced a very interesting geometric notion "higher order paratangent bundle" $\tau_{N}^{d}(X)$ to this problem, generalizing Glaeser's paratangent bundle. This is a variant of higher order tangent bundle of $X$. Further, they associated to every function $f: X \longrightarrow \mathbb{R}$, a subbundle $\nabla_{N}^{d} f \subset \tau_{N}^{d}(X) \times \mathbb{R}(d, N \in \mathbb{N})$ over $X$. In these constructions of $\tau_{N}^{d}(X)$ and $\nabla_{N}^{d} f$, they used a set operation found by Glaeser (see 93). They posed a general conjecture on the extension problem as follows.

(*) A function $f$ is $C^{d}$ extendable if and only if $\nabla_{N}^{d} f$ is the graph of a mapping

Date: November 21, 2018. 
of $\tau_{N}^{d}(X)$ into $\mathbb{R}$ for a suitable $N$.

They proved that, for the closure $X$ of an open subset of a regular submanifold of a Euclidean domain, $\tau_{N}^{d}(X)$ coincides with the full higher order tangent bundle of the submanifold on $X$ and that the conjecture $(*)$ is affirmative for such $X$. Further, they have obtained a positive result for the important case of compact subanalytic sets.

We call the second problem flatness problem since uniqueness up to order $r$ of Taylor expansion at $\xi \in X$ is assured by controlling $r$-flatness by the values on $X$. This problem was first considered by Spallek $\underline{S}$. The present author [ developed fundamental properties of "Spallek function", an invariant defined for the germ $X_{\xi}$ which measures the efficiency of flatness control of functions at $\xi$ by their values on $X$.

Since Whitney's works, it has been widely known that the theory of smooth functions is closely related to the interpolation theory (e.g. $\mathrm{W}_{2}, \mathrm{G}_{2}, \mathrm{~K}, \mathrm{MM}$, $\left.[\mathrm{S}], \mathrm{BMP}_{2}, \mathrm{I}_{]}\right)$. The reason is that differential properties are not punctual but "molecular" (Glaeser) as seen in the bi-punctual inequality used to define Whitney function $\left(\left[\mathrm{W}_{1}\right)\right.$. Glaeser $\mathrm{G}_{2}$ proposed two methods of application of interpolation, Lagrange interpolation and "interpolation schemes" to treat differential properties. He put emphasis on the latter. But we adopt the former method in this paper. Following Glaeser, we treat interpolations with $(n+d) ! / n ! d$ ! nodes for the problems of $C^{d}$ functions on $\mathbb{R}^{n}$. The most important point is that "Vandermonde matrix" appears in the matrix representation of Taylor expansion (see the proofs of 4.1 and 6.2). We measure the decrease of the Vandermonde determinants of accumulating nodal sets.

In 4.1 we show the following. Suppose that $X \subset \mathbb{R}^{n}$ includes nodal sets accumulating to a point and that their Vandermonde determinants are not rapidly decreasing relative to their diameters. Then $X$ has the full higher order paratangent spaces at the accumulating point. Remember that if $X$ has full higher order paratangent spaces at any point of $X$, the conjecture $(*)$ is valid for such $X$ (see $\left[\mathrm{BMP}_{2}\right]$. Many classical fractal sets, such as Cantor set, Koch curve, Sierpinski gasket and Menger sponge, satisfy this condition (see [5.2).

As for flatness, we need a more quantitative argument. We give a sufficient condition 6.2 for sets $X$ to control flatness of functions at a point of $X$ (see [6.2) . This is nothing but a sharpened form of Spallek's theorem.

We consider that our study still leaves a major portion of the extension problem open. We treat only rather easy phenomena in the following sense. A set satisfying the condition in the main theorem 4.1 has always the full higher order paratangent space at the accumulating point. The construction of the paratangent bundle requires repetition of Glaeser operations in general (cf. $\left.\mathrm{BMP}_{2}, 1.8\right)$. In 
contrast, the sets treated in 4.1 call for it only once. By the result $\mathrm{BMP}_{2}, 1.3$, it might be inevitable to assume the graphic condition of $\tau_{N}^{e}(f)$ with $e>d$ for $C^{d}$ extension in general.

In the case of the flatness problem, it is interesting to analyse the growth of Spallek functions (cf. [I] $, 2.9,3.6,4.4$ ). But we have no idea to connect our present method to observe them.

This work was partly done during the stay at Universite des Science et Technologies de Lille. The author wish to express his sincere thanks for the courtesy and for the helpful discussions with the participants of Séminaire d'Analyse Complexe et Différentielle of Lille, in particular to Professor Ann-Marie Chollet. He also would like to thank the participants of Seminar of Functions of Complex Variables of Kyoto.

\section{Multivariate Lagrange interpolation}

Let us recall some elementary facts on Lagrange interpolation in $\mathbb{R}^{n}$. In the following, functions are $\mathbb{R}$ valued and linearity is over $\mathbb{R}$.

Proposition 1.1. Let $A$ be a subset of $\mathbb{R}^{n}$ of $N$ distinct points and $f_{1}, \ldots, f_{N}$ be functions defined on $A$. Then the following conditions are equivalent.

(1) A is not contained in the vanishing locus of any non-trivial linear combination of $f_{1}, \ldots, f_{N}$.

(2) For any set of values prescribed at each point of $A$, there exists at most one linear combination of $f_{1}, \ldots, f_{N}$ which takes these values at each point of $A$.

(3) For any set of values prescribed at each point of $A$, there exists at least one linear combination of $f_{1}, \ldots, f_{N}$ which takes these values at each point of $A$.

Proof. Let $V:=\left(f_{i}\left(\boldsymbol{a}_{j}\right)\right)$ denote the square matrix of the values of $f_{i}$ at points $\boldsymbol{a}_{1}, \ldots, \boldsymbol{a}_{N}$. Then, (1) and (2) are equivalent to the condition that the row vectors of $V$ are independent. The condition (3) is equivalent to saying that the vectors generate the whole $N$ dimensional space. Since $V$ is square, (1), (2), (3) are all equivalent to the condition that $V$ is regular.

Proposition 1.2. If $S \subset \mathbb{R}^{n}$ is not contained in the vanishing locus of any nontrivial linear combination of $f_{1}, \ldots, f_{M}$, there exists $A \subset S$ such that $\# A=M$ and $A$ is not contained in such a locus either.

Proof. Let $W:=\left(f_{i}(\boldsymbol{s})\right)_{i=1, \ldots, M ;} \boldsymbol{s \in S}$ denote the (possibly infinite) matrix of the values of $f_{i}$ at points $s \in S$. The rows of $W$ are linearly independent by our assumption. Hence, there is an $M \times M$ regular minor matrix. Then the set $A$ of the points corresponding to the columns of the minor satisfies the condition. 


\section{Polynomial interpolation}

For a subset $A$ of $\mathbb{R}^{n}$, let $\operatorname{Hdeg}(A)$ denote the minimum of the degrees of non-zero polynomials vanishing on $A$. If there is no such polynomial, we put $\operatorname{Hdeg}(A)=\infty$. The dimension of the vector space of homogeneous polynomials in $n+1$ variables of degree $d$ coincides with that of the vector space of polynomials in $n$ variables of degree less than or equal to $d$. We express it by

$$
N(n, d)=N(d, n):=\left(\begin{array}{c}
n+d \\
d
\end{array}\right)=\frac{(n+d) !}{n ! d !} .
$$

Proposition 2.1. If $A \subset \mathbb{R}^{n}$ and $\operatorname{Hdeg}(A) \geq d+1$, then $\# A \geq N(n, d)$.

Proof. Consider the vectors of dimension \#A whose components are the values of a monomial of degree less than or equal to $d$ at points of $A$. Such vectors are $N(n, d)$ in number. If $\# A<N(n, d)$, these vectors are linearly dependent. This implies that some non-trivial linear combination of the monomials vanishes at each point of $A$. This contradicts the assumption $\operatorname{Hdeg}(A) \geq d+1$.

Let us take the set of multi-indices

$$
\begin{gathered}
I=I(n, d):=\left\{\boldsymbol{i}:=\left(i_{1}, \ldots, i_{n}\right) \in\{0, \ldots, d\}^{n}:|\boldsymbol{i}| \leq d\right\} \\
\left(|\boldsymbol{i}|:=i_{1}+\cdots+i_{n}\right)
\end{gathered}
$$

and express the monomials and the derivatives in $\boldsymbol{x}:=\left(x_{1}, \ldots, x_{n}\right)$ as follows:

$$
\begin{gathered}
\boldsymbol{x}^{\boldsymbol{i}}:=x_{1}^{i_{1}} \cdots x_{n}^{i_{n}} \quad\left(\boldsymbol{i}:=\left(i_{1}, \ldots, i_{n}\right) \in I\right), \\
f^{(\boldsymbol{p})}:=\frac{\partial^{|\boldsymbol{p}|} f}{\partial^{p_{1}} x_{1} \cdots \partial^{p_{n}} x_{n}} \quad\left(\boldsymbol{p}:=\left(p_{1}, \ldots, p_{n}\right) \in I\right) .
\end{gathered}
$$

Lemma 2.2. The sum of the degrees of all monomials in $n$ variables of degrees less than or equal to $d$ is equal to the following numbers.

$$
\begin{aligned}
\sum_{\boldsymbol{p} \in I(n, d)}|\boldsymbol{p}| & =n \sum_{i=1}^{d} i \cdot N(n-1, d-i)=\sum_{i=1}^{d} i \cdot N(n-1, i) \\
& =n \sum_{i=0}^{d-1} N(n, i)=n \cdot N(n+1, d-1) .
\end{aligned}
$$

Proof. Let $x_{1}, \ldots, x_{n}$ be the variables. Let $S_{k}(k=1,2, \ldots, 5)$ denote the $k$-th expression in the equality above. The first expression $S_{1}$ is just the quantity mentioned at the top of the lemma. The expression $S_{2}$ is obtained by counting the degrees in $x_{p}$ separately for each $p$. The summand is the sums for the terms of degree just $i$ in $x_{p}$. The expression $N(n-1, d-i)$ denotes the number of such terms, of degree equal to or smaller than $d-i$ in the variables other than $x_{p}$. The preceding multiplier $n$ is the number of the choice of $p$. The summand of $S_{3}$ is 
equal to the product of the degree $i$ and the number of the monomial bases in $n$ variables of degree just $i$. The equality $S_{3}=S_{4}$ follows from

$$
\sum_{i=1}^{d} i \cdot N(n-1, i)=\sum_{i=1}^{d} \frac{(n+i-1) !}{(n-1) !(i-1) !}=n \sum_{i=0}^{d-1} N(n, i) .
$$

The last equality $S_{4}=S_{5}$ follows from the obvious equality

$$
N(n, i)=N(n+1, i)-N(n+1, i-1) .
$$

Suppose that $A$ is a set of $N(n, d)$ distinct points in $\mathbb{R}^{n}$ indexed as

$$
A:=\left\{\boldsymbol{a}_{\boldsymbol{i}}:=\left(a_{i_{1}}, \ldots, a_{i_{n}}\right): \boldsymbol{i} \in I\right\} .
$$

Fixing an ordering of $I$, we obtain an $N(n, d) \times N(n, d)$ matrix

$$
V(A):=V\left(\boldsymbol{a}_{\boldsymbol{i}}: \boldsymbol{i} \in I\right):=\left(\boldsymbol{a}_{\boldsymbol{i}}^{\boldsymbol{j}}\right)=\left(a_{i_{1}}^{j_{1}} \cdots a_{i_{n}}^{j_{n}}\right),
$$

where $\boldsymbol{i} \in I$ are multi-suffixes seen as the row indices and $\boldsymbol{j} \in I$ are multi-exponents seen as the column indices. $V(A)$ is called the $n$-dimensional Vandermonde matrix of $A$ (cf. [AS]). This has the following properties.

Proposition 2.3. Let $A:=\left\{\boldsymbol{a}_{\boldsymbol{i}}: \boldsymbol{i} \in I\right\} \subset \mathbb{R}^{n}$ be a subset of $N(n, d)$ distinct points.

(1) Det $V(A)$ is homogeneous of degree $N(n+1, d-1)$ with respect to the $p$-th coordinates of $\boldsymbol{a}_{\boldsymbol{i}}(\boldsymbol{i} \in I)$ for each fixed $p=1, \ldots, n$.

(2) Let $\varphi: \mathbb{R}^{n} \longrightarrow \mathbb{R}^{n}$ be a linear transformation expressed by a matrix $P$. Then

$$
\text { Det } V(\varphi(A))=(\operatorname{Det} P)^{N(n+1, d-1)} \operatorname{Det} V(A) \text {. }
$$

(3) Det $V(A)$ is an invariant of translations i.e.

$$
\text { Det } V(A-\boldsymbol{v})=\operatorname{Det} V(A) \quad\left(\boldsymbol{v} \in \mathbb{R}^{n}\right) .
$$

Proof.

(1) This follows from $2.2\left(S_{1}=S_{5}\right.$ in the proof).

(2) The general linear group $G L(n, \mathbb{R})$ is generated by the transformations of the following forms.

(a) $y_{1}=x_{1}, \ldots, y_{p}=x_{p}+\lambda x_{q}, \ldots, y_{n}=x_{n} \quad(p \neq q)$,

(b) $y_{1}=x_{1}, \ldots, y_{p}=\lambda x_{p}, \ldots, y_{n}=x_{n}(\lambda \neq 0)$.

In the case of (a), Det $P=1$ and Det $V(\varphi(A))=$ Det $V(A)$. In the case of (b), Det $P=\lambda$. Since Det $V(A)$ is homogeneous of degree $N(n+1, d-1)$ with respect to the $p$-th coordinates of $\boldsymbol{a}_{\boldsymbol{i}}(\boldsymbol{i} \in I)$, we have

$$
\begin{gathered}
\text { Det } V(\varphi(A))=\lambda^{N(n+1, d-1)} \operatorname{Det} V(A) \\
=(\operatorname{Det} P)^{N(n+1, d-1)} \operatorname{Det} V(A) .
\end{gathered}
$$


These prove the equalities.

(3) In view of (2), we have only to prove the invariance with respect to the transformations in $x_{1}$ direction, which follows in the same way as the case (a) above.

Remark 2.4. We call $\varphi: \mathbb{R}^{n} \longrightarrow \mathbb{R}^{n}$ affine if it is a composition of an invertible linear transformation $\varphi^{\prime}$ after (or before) a translation $\varphi^{\prime \prime}$. If $\varphi$ is an affine transformation, the determinant of its linear part $\varphi^{\prime}$ is the ratio of the signed volumes of $\varphi(B)$ and $B$ for any measurable subset $B$. Thus the proposition above implies that the quotient $V(\varphi(A)) / V(A)$ is expressed as the $N(n+1, d-1)$-th power of the ratio of the signed volumes of $\varphi(B)$ and $B$.

Applying 1.1] and 2.1] to the monomials $\boldsymbol{x}^{j}$, we have the following.

Proposition 2.5. Suppose that $A$ is a set of $N(n, d)$ distinct points in $\mathbb{R}^{n}$ with $n, d \in \mathbb{N}$. Then $\operatorname{Hdeg}(A) \leq d+1$ and the following conditions are equivalent.

(1) $\operatorname{Hdeg}(A)=d+1$.

(2) Det $V(A) \neq 0$.

(3) There is a unique polynomial of degree at most $d$ which takes the set of values prescribed at each point of $A$.

The author knows the following geometric interpretations of these conditions.

(1) The case $n=1$ is well known: Det $V(A) \neq 0$.

(2) The case $d=1$ implies that $n+1$ points $\boldsymbol{a}_{i}:=\left(a_{i 1}, \ldots, a_{i n}\right)(i=0, \ldots, n)$ in $\mathbb{R}^{n}$ are contained in a hyperplane if and only if

$$
\left|\begin{array}{cccc}
a_{01} & \ldots & a_{0 n} & 1 \\
\ldots \ldots & \ldots & \ldots & \ldots \\
a_{n 1} & \ldots & a_{n n} & 1
\end{array}\right|=0 .
$$

(3) Suppose that $n=d=2$. Let $\boldsymbol{b}_{1}, \boldsymbol{b}_{2}, \boldsymbol{b}_{3}$ denote the intersection points of the line joining $\boldsymbol{a}_{110}$ and $\boldsymbol{a}_{020}$ and the line joining $\boldsymbol{a}_{101}$ and $\boldsymbol{a}_{002}$; the line joining $\boldsymbol{a}_{011}$ and $\boldsymbol{a}_{002}$ and the line joining $\boldsymbol{a}_{110}$ and $\boldsymbol{a}_{200}$; the line joining $\boldsymbol{a}_{101}$ and $\boldsymbol{a}_{200}$ and the line joining $\boldsymbol{a}_{011}$ and $\boldsymbol{a}_{020}$ respectively. By Pascal's theorem, (1) in the proposition is equivalent to the condition that $\boldsymbol{b}_{1}, \boldsymbol{b}_{2}, \boldsymbol{b}_{3}$ are not contained in a line.

(4) A sufficient condition for (2) for general $n, d$ is given in $[\mathrm{AS}$, 3.6. A convenient version of this condition is given in [1]. (The author does not know whether this convenient version loses generality or not in comparison to AS], 3.6.) These assure that a general set $A$ of $N(n, d)$ points has nonvanishing $\operatorname{Det} V(A)$. 


\section{Higher ORDER PARATANGENT BUNDLE}

Bierstone, Milman and Pawłucki $\mathrm{BMP}_{2}$ have defined the higher order paratangent bundle $\tau_{N}^{d}(X)$ of order $d$ for a subset $X$ of a Euclidean space (or of a manifold), generalising Glaeser's paratangent bundle. We briefly describe the necessary part here. Note that we adopt the general definition of $\tau_{N, \boldsymbol{x}}^{d}(X)$ explained in the last section of $\left[\mathrm{BMP}_{2}\right.$, whereas they used only the case $N=1$ in the main part of the paper. Necessity of using larger $N$ was already pointed out and related lemmas were prepared by them.

Let $X$ be a metric space and $V$ a finite dimensional $\mathbb{R}$ vector space. We call a subset $E \subset X \times V$ a bundle (of subspaces of $V$ ) over $X$ if the fibres $E_{\boldsymbol{a}}:=\{v$ : $(\boldsymbol{a}, v) \in E\}$ are linear subspaces of $V$.

Let $X$ be a subset of a Euclidean space $\mathbb{R}^{n}$. Let $\mathcal{P}_{d}$ be the $\mathbb{R}$ vector space of polynomials of degree equal to or less than $d$ and $\mathcal{P}_{d}^{*}$ its dual vector space. Let us denote assignment of the value $(-1)^{|\boldsymbol{p}|}\left(\partial^{|\boldsymbol{p}|} f / \partial \boldsymbol{x}^{\boldsymbol{p}}\right)(\boldsymbol{a})$ to $f \in \mathcal{P}_{d}$ by $\delta_{\boldsymbol{a}}^{(\boldsymbol{p})}$. This expresses the derivative of the Dirac delta function of order $\boldsymbol{p}$ with $|\boldsymbol{p}| \leq d$. We adopt this symbol because of notational simplicity. But take care that it has different properties according to $d$. For example, $\delta_{a}=\delta_{b}$ always holds in $\mathcal{P}_{0}^{*}$ but not in $\mathcal{P}_{1}^{*}$. We have $\delta_{a}=\delta_{b}-\sum\left(a_{i}-b_{i}\right) \delta_{b}^{\left(e_{i}\right)}$ and $\delta_{a}^{\left(e_{i}\right)}=\delta_{b}^{\left(e_{i}\right)}$ in $\mathcal{P}_{1}^{*}$, where $e_{i}$ is the multi-index whose $i$-th component is 1 and other components are 0 . In general, the derivatives $(-1)^{|\boldsymbol{p}|} \delta_{\boldsymbol{a}}^{(\boldsymbol{p})} / \boldsymbol{p} !\left(\boldsymbol{p}:=p_{1} !, \ldots, p_{n}\right.$ !, $\left.|\boldsymbol{p}| \leq d\right)$ form the dual basis of $\left\{(\boldsymbol{x}-\boldsymbol{a})^{\boldsymbol{p}}:|\boldsymbol{p}| \leq d\right\}$ and all the derivatives of $\delta_{\boldsymbol{a}}$ are expressed by those of $\delta_{\boldsymbol{b}}$ in $\mathcal{P}_{d}^{*}$ (see the first equality in the proof of 4.1).

Let us put

$$
E_{0}:=\left\{\left(\boldsymbol{a}, \lambda \delta_{\boldsymbol{a}}\right): \boldsymbol{a} \in X, \lambda \in \mathbb{R}\right\} .
$$

We define $E_{k}$ inductively as follows. If $E_{k}$ is defined, put

$$
\begin{aligned}
& \Delta E_{k}:=\left\{\left(\boldsymbol{a}_{0}, \ldots, \boldsymbol{a}_{N}, \xi_{0}+\cdots+\xi_{N}\right): \boldsymbol{a}_{i} \in X, \xi_{i} \in E_{k, \boldsymbol{a}_{i}},\right. \\
& \left.\left|\boldsymbol{a}_{i}-\boldsymbol{a}_{0}\right|^{d-|\alpha|}\left|\xi_{i}\left(\left(\boldsymbol{x}-\boldsymbol{a}_{i}\right)^{\alpha}\right)\right| \leq 1(|\alpha| \leq d, 0 \leq i \leq N)\right\}
\end{aligned}
$$

and

$$
E_{k}^{\prime}:=\pi\left(\overline{\Delta E_{k}} \cap\left\{(\boldsymbol{a}, \ldots, \boldsymbol{a}, \xi): \boldsymbol{a} \in X, \xi \in \mathcal{P}_{d}^{*}\right\}\right),
$$

where $\pi: X \times \cdots \times X \times \mathcal{P}_{d}^{*} \longrightarrow X \times \mathcal{P}_{d}^{*}$ denotes the canonical projection onto the first $X$ times $\mathcal{P}_{d}^{*}$. The intersection of the closure of $\Delta E_{k}$ and the diagonal coincides with the set of all the limiting points of

$$
\left(\boldsymbol{a}_{0}, \ldots, \boldsymbol{a}_{N}, \xi_{0}+\cdots+\xi_{N}\right) \in \Delta E_{k}
$$

when $\boldsymbol{a}_{0}, \ldots, \boldsymbol{a}_{N}$ approach to $\boldsymbol{a}$. Finally we put

$$
E_{k+1}:=\bigcup_{\boldsymbol{a} \in X}\left(\{\boldsymbol{a}\} \times \operatorname{Span} E_{k, \boldsymbol{a}}^{\prime}\right) \subset X \times \mathcal{P}_{d}^{*},
$$


where Span $E_{k}^{\prime}$ denotes the linear span of $E_{k}^{\prime}$ in the fibre. The procedure of obtaining $E_{k+1}$ from $E_{k}$ is an example of Glaeser operation in $\mathrm{BMP}_{2}$. The sequence $E_{1} \subset E_{2} \subset E_{3} \subset \cdots$ stabilizes and we have $E_{k}=E_{2 \operatorname{dim} \mathcal{P}_{d}^{*}}\left(k \geq 2 \operatorname{dim} \mathcal{P}_{d}^{*}\right)$ as a general property of Glaeser operation $\left(\mathrm{G}_{1} ; \mathrm{BMP}_{2}, 3.3\right)$. This saturation $\tau_{N}^{d}(X):=E_{2 \operatorname{dim} \mathcal{P}_{d}^{*}}$ is called the paratangent bundle of order $d$ of $X\left(\mathrm{BMP}_{2}\right)$. This is a closed subbundle of $X \times \mathcal{P}_{d}^{*}$ in the obvious sense. It is known that, for a subbundle (of subspaces), closedness is equivalent to upper semi-continuity of inclusion ([C], p.67). Let us call the fibre $\tau_{N, \boldsymbol{a}}^{d}(X)$ the paratangent space of order $d$ of $X$ at $\boldsymbol{a}$. Glaeser's (linearized) paratangent bundle is isomorphic to $\tau_{1}^{1}(X)$.

Remark 3.1. We can replace the control condition

$$
\left|\boldsymbol{a}_{i}-\boldsymbol{a}_{0}\right|^{d-|\alpha|}\left|\xi_{i}\left(\left(\boldsymbol{x}-\boldsymbol{a}_{i}\right)^{\alpha}\right)\right| \leq 1 \quad(N \leq r)
$$

by

or by

$$
\left|\boldsymbol{a}_{i}-\boldsymbol{a}_{0}\right|^{d-|\alpha|}\left|\xi_{i}\left(\left(\boldsymbol{x}-\boldsymbol{a}_{i}\right)^{\alpha}\right)\right| \leq c
$$

$$
\left|\boldsymbol{a}_{i}-\boldsymbol{a}_{0}\right|^{d-|\alpha|}\left|\xi_{i}\left(\left(\boldsymbol{x}-\boldsymbol{a}_{0}\right)^{\alpha}\right)\right| \leq c
$$

with any $c>0$ independent of $i$ and $\alpha\left(\mathrm{BMP}_{2}, \S 5\right)$. This control condition is used to prove the easy half of the conjecture below (cf. (4.7), (4.16), (4.17) of $\left[\mathrm{BMP}_{2}\right)$. Hence the control condition seems to endow $\tau_{N, \boldsymbol{a}}^{d}(X)$ a character peculiar to class $C^{d}$.

Now we describe the construction of $\nabla_{N}^{d} f$ in order to have its image, although we do not use its explicit form later. Consider a continuous function $f: X \longrightarrow \mathbb{R}$ and the bundle

$$
\Phi_{0}:=\left\{\left(\boldsymbol{a}, \lambda \delta_{\boldsymbol{a}}, \lambda f(\boldsymbol{a})\right): \boldsymbol{a} \in X, \lambda \in \mathbb{R}\right\} \subset X \times \mathcal{P}_{d}^{*} \times \mathbb{R}
$$

over $X$. If $\Phi_{k}$ is defined, put

$$
\begin{gathered}
\Delta \Phi_{k}:=\left\{\left(\boldsymbol{a}_{0}, \ldots, \boldsymbol{a}_{N}, \xi_{0}+\cdots+\xi_{N}, \lambda_{0}+\cdots+\lambda_{N}\right):\right. \\
\boldsymbol{a}_{i} \in X,\left(\xi_{i}, \lambda_{i}\right) \in \Phi_{k, a_{i}},\left|\boldsymbol{a}_{i}-\boldsymbol{a}_{0}\right|^{d-|\alpha|}\left|\xi_{i}\left(\left(\boldsymbol{x}-\boldsymbol{a}_{i}\right)^{\alpha}\right)\right| \leq 1 \\
(|\alpha| \leq d, 0 \leq i \leq N)\}
\end{gathered}
$$

and

$$
\Phi_{k}^{\prime}:=\pi\left(\overline{\Delta \Phi_{k}} \cap\left\{(\boldsymbol{a}, \ldots, \boldsymbol{a}, \xi, \lambda): \boldsymbol{a} \in X, \xi \in \mathcal{P}_{d}^{*}, \lambda \in \mathbb{R}\right\}\right)
$$

where

$$
\pi: X \times \cdots \times X \times \mathcal{P}_{d}^{*} \times \mathbb{R} \longrightarrow X \times \mathcal{P}_{d}^{*} \times \mathbb{R}
$$

denotes the canonical projection onto the first $X$ times $\mathcal{P}_{d}^{*} \times \mathbb{R}$. Finally we put

$$
\Phi_{k+1}:=\bigcup_{\boldsymbol{a} \in X}\left(\{\boldsymbol{a}\} \times \operatorname{Span} \Phi_{k, \boldsymbol{a}}^{\prime}\right) \subset X \times \mathcal{P}_{d}^{*} \times \mathbb{R} .
$$


Since the extension $\Phi_{k} \subset \Phi_{k+1}$ is also a Glaeser operation, the sequence $\Phi_{0} \subset \Phi_{1} \subset$ $\Phi_{2} \subset \ldots$ stabilizes for $i \geq 2 \operatorname{dim}\left(\mathcal{P}_{d}^{*} \times \mathbb{R}\right)\left(\mathrm{G}_{1}, \mathrm{BMP}_{2}, 3.3\right)$ and the saturation is denoted by $\nabla_{N}^{d} f$.

Conjecture 3.2 (Bierstone-Milman-Pawłucki). Let $X$ be a closed subset of $\mathbb{R}^{n}$. Then there exists $N \in \mathbb{N}$ such that a continuous function $f: X \longrightarrow \mathbb{R}$ can be extended to a $C^{d}$ function if and only if $\nabla_{N}^{d} f \subset \tau_{N}^{d}(X) \times \mathcal{P}_{d}^{*}$ is a graph of a map of $\tau_{N}^{d}(X)$ into $\mathbb{R}$.

Only-if part was proved by themselves $\left(\mathrm{BMP}_{2}, 4.17\right)$. They proved if part when $X$ is the closure of an open subset of a regular submanifold. The paratangent bundle $\nabla_{N}^{d} f$ with $N=1$ is sufficient for their proof. We prove a sharper form of this result in the next section. They also proved the case of compact subanalytic sets with some loss of differentiability using their deep results $\mathrm{BMP}_{1}$ on composite functions.

Remark 3.3. Suppose that $X$ is a closed subset of $\mathbb{R}^{n}$ and $Y$ a dense subset of $X$. If $\tau_{N, \xi}^{d}(X)=\mathcal{P}_{d}^{*}$ for any $\xi \in Y$, then $\tau_{N, \xi}^{d}(X)=\mathcal{P}_{d}^{*}$ for any $\xi \in X$ by the closedness

of $\tau_{N, \xi}^{d}(X)$. Then the conjecture $\mathbf{3 . 2}$ is affirmative for such an $X$ by $\mathrm{BMP}_{2}$, Proof of 4.20 .

Remark 3.4. $\left(\mathrm{BMP}_{2}, 4.23\right)$ Suppose that $X \subset M \subset \mathbb{R}^{n}$, where $M$ an $m$ dimensional regular submanifold of $\mathbb{R}^{n}$ and that $\tau_{N, \xi}^{d}(X)=\tau_{N, \xi}^{d}(M)$ for any $\xi \in X$. Then the conjecture $\mathbf{3 . 2}$ is affirmative for such an $X$.

\section{Set Germs With FUlL Higher order PARATANGENT SPACES}

Theorem 4.1. Let $\left\{r_{k}\right\} \subset \mathbb{R}$ be a positive sequence and $A_{k}:=\left\{\boldsymbol{a}_{0}^{k}, \ldots, \boldsymbol{a}_{N}^{k}\right\} \subset \mathbb{R}^{n}$ sets of $N(n, d)=N+1$ distinct points $(k \in \mathbb{N})$. Suppose that

(1) $A_{k}$ is contained in the closed ball of radius $r_{k}$ centred at $\boldsymbol{a}_{0}^{k}$;

(2) $\lim _{k \rightarrow \infty} \boldsymbol{a}_{0}^{k}=\boldsymbol{\xi}$

(3) $\lim _{k \rightarrow \infty} r_{k}=0$;

(4) there exists $c>0$ independent of $k$ such that

$$
\left|\operatorname{Det} V\left(A_{k}\right)\right| \geq c \cdot r_{k}^{n \cdot N(n+1, d-1)} \quad(k \in \mathbb{N}) \text {. }
$$

If $X$ is a closed subset including $\bigcup A_{k}$, then $\tau_{N, \boldsymbol{\xi}}^{d}(X)=\mathcal{P}_{d}^{*}$. 

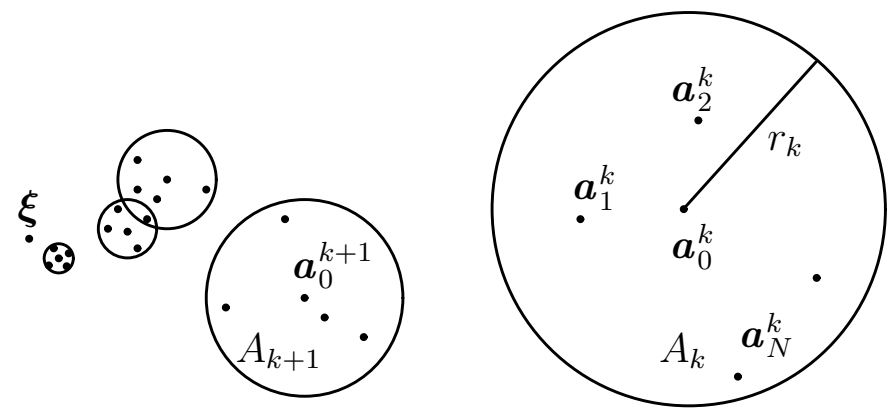

Remark 4.2. In view of 2.5, the condition (4) implies that the points of $A_{k}$ are algebraically in general position in the balls of (1) uniformly with respect to $k$. If the interior of $X$ is adherent to 0 , this condition is satisfied. Hence, by $\mathrm{BMP}_{2}$, Proof of 4.20 (or 3.3 with $Y=X$ ), we see that our theorem is an improvement of $\mathrm{BMP}_{2}$, 4.19: balls are replaced by the sets $A_{k}$ of $N+1$ points.

Proof of 4.1. Let $\boldsymbol{p}_{0}, \ldots, \boldsymbol{p}_{N}$ denote the elements of $I(n, d)$. If $f$ is a polynomial of degree $d$, we have

$$
V\left(A_{k}-\boldsymbol{a}_{\mathbf{0}}^{k}\right)\left(\begin{array}{c}
\frac{f^{\left(\boldsymbol{p}_{0}\right)}\left(\boldsymbol{a}_{0}^{k}\right)}{\boldsymbol{p}_{0} !} \\
\vdots \\
\frac{f^{\left(\boldsymbol{p}_{N}\right)}\left(\boldsymbol{a}_{0}^{k}\right)}{\boldsymbol{p}_{N} !}
\end{array}\right)=\left(\begin{array}{c}
f\left(\boldsymbol{a}_{0}^{k}\right) \\
\vdots \\
f\left(\boldsymbol{a}_{N}^{k}\right)
\end{array}\right) .
$$

Namely, the Dirac deltas $\delta_{\boldsymbol{a}_{0}^{k}}, \ldots, \delta_{\boldsymbol{a}_{N}^{k}}$ are expressed in terms of the higher order derivatives $\delta_{\boldsymbol{a}_{0}^{k}}^{\left(\boldsymbol{p}_{0}\right)}, \ldots, \delta_{\boldsymbol{a}_{0}^{k}}^{\left(\boldsymbol{p}_{N}\right)}$ of the Dirac delta at $\boldsymbol{a}_{0}^{k}$ in $\mathcal{P}_{d}^{*}$.

Since the Vandermonde determinant does not vanish, $\delta_{\boldsymbol{a}_{0}^{k}}^{\left(\boldsymbol{p}_{\boldsymbol{i}}\right)}$ are spanned by $\delta_{\boldsymbol{a}_{1}^{k}}, \ldots, \delta_{\boldsymbol{a}_{N}^{k}}$. All the elements of $V\left(A_{k}-\boldsymbol{a}_{\mathbf{0}}^{k}\right)$ with column index $i$ are homogeneous polynomials of degree $\left|\boldsymbol{p}_{i}\right|$ in all the components of all $\boldsymbol{a}_{i}-\boldsymbol{a}_{0}$ and the Vandermonde determinant Det $V\left(A_{k}-\boldsymbol{a}_{\mathbf{0}}^{k}\right)$ is a homogeneous polynomial of degree $n \cdot N(n+1, d-1)$ in them. Then the elements with row index $i$ of the cofactor matrix (Det $V\left(A_{k}-\right.$ $\left.\left.\boldsymbol{a}_{\mathbf{0}}^{k}\right)\right) V\left(A_{k}-\boldsymbol{a}_{\mathbf{0}}^{k}\right)^{-1}$ are homogeneous of degree

$$
\sum_{j \neq i}\left|\boldsymbol{p}_{j}\right|=\sum_{j}\left|\boldsymbol{p}_{j}\right|-\left|\boldsymbol{p}_{i}\right|=n \cdot N(n+1, d-1)-\left|\boldsymbol{p}_{i}\right|
$$

in them. Here, the last equality follows from 2.2. Applying the condition (4) and 2.2. we see that all the elements of $V\left(A_{k}-\boldsymbol{a}_{\mathbf{0}}^{k}\right)^{-1}$ with row index $i$ are majorized by a constant multiple of $r_{k}^{-\left|\boldsymbol{p}_{i}\right|} \leq r_{k}^{-d}$. Hence the control conditions for the coefficients of $\delta_{\boldsymbol{a}_{0}^{k}}$ in the construction of $\tau_{N, \mathbf{0}}^{d}(X)$ are satisfied. Then $\delta_{\mathbf{0}}^{\left(\boldsymbol{p}_{j}\right)} \in \tau_{N, \mathbf{0}}^{d}(X)$ follows as 
their limits.

\section{Paratangent Bundles of SElF-Similar SETS}

First we recall the definition of self-similar set. The readers can refer to $\mathrm{F}$ and [YHK] for further explanation. A map $\varphi: \mathbb{R}^{n} \longrightarrow \mathbb{R}^{n}$ is called a contraction if there exists $K \in(0,1)$ such that

$$
\|\varphi(\boldsymbol{x})-\varphi(\boldsymbol{y})\| \leq K \cdot\|\boldsymbol{x}-\boldsymbol{y}\| \quad\left(\boldsymbol{x}, \boldsymbol{y} \in \mathbb{R}^{n}\right) .
$$

If a finite set $\varphi_{1}, \ldots, \varphi_{p}: \mathbb{R}^{n} \longrightarrow \mathbb{R}^{n}$ of contractions of $\mathbb{R}^{n}$ is given, there exists a unique non-empty compact set $S \subset \mathbb{R}^{n}$ such that $S=\bigcup_{i=1}^{p} \varphi_{i}(S)$. Such an $S$ is called the attractor or the invariant set of $\varphi_{1}, \ldots, \varphi_{p}$. In particular, every contraction $\varphi$ of $\mathbb{R}^{n}$ has a unique fixed point $F(\varphi)$. The following is known as Williams' formula. For the attractor of $\varphi_{1}, \ldots, \varphi_{p}$, we have

$$
S=\longdiv { \bigcup F ( \varphi _ { i _ { 1 } } \circ \cdots \circ \varphi _ { i _ { q } } ) }
$$

where $\left(i_{1}, \ldots, i_{q}\right)$ runs over all finite sequences of elements of $\{1, \ldots, p\}$. Let us call an affine transformation a similarity transformation if it preserves the angle of every ordered triplet of points. If $\varphi$ is a similarity transformation, there exists $\lambda \in(0, \infty)$ such that

$$
\|\varphi(\boldsymbol{x})-\varphi(\boldsymbol{y})\|=\lambda \cdot\|\boldsymbol{x}-\boldsymbol{y}\| \quad\left(\boldsymbol{x}, \boldsymbol{y} \in \mathbb{R}^{n}\right)
$$

We call $\lambda$ the similarity ratio of $\varphi$. An attractor of $\varphi_{1}, \ldots, \varphi_{p}(p \geq 2)$ is called self-similar, if all $\varphi_{i}$ are similarity transformations (see $[\mathrm{F}]$ ). (Often more general attractors are called self-similar (see YHK, p.18).) The next lemma is almost immediate from 2.4

Lemma 5.1. Let $A$ be a point set with $\# A=N+1=N(n, d)$ and $B$ another point set similar to $A$. Then we have

$$
\frac{\operatorname{Det} V(A)}{\delta(A)^{n \cdot N(n+1, d-1)}}=\frac{\operatorname{Det} V(B)}{\delta(B)^{n \cdot N(n+1, d-1)}},
$$

where $\delta$ denotes the diameter.

Theorem 5.2. For any $d \in \mathbb{N}$, we define $N$ by $N(n, d)=N+1$. Let $X \subset \mathbb{R}^{n}$ be a self-similar subset with $\operatorname{Hdeg}(X) \geq d+1$. Then $\tau_{N, s}^{d}(X)=\mathcal{P}_{d}^{*}$ for any $s \in X$. 
Proof. Suppose that $X$ is defined by contracting similarity transformations $\varphi_{1}, \ldots, \varphi_{p}$. By 3.3, we have only to prove that $\tau_{N, s}^{d}(X)=\mathcal{P}_{d}^{*}$ for points $s$ of a dense subset of $X$. Then, by Williams' formula, we may assume that $s$ is the fixed point of $\varphi_{i_{1}} \circ \cdots \circ \varphi_{i_{k}}$, i.e. $s=F\left(\varphi_{i_{1}} \circ \cdots \circ \varphi_{i_{k}}\right)$.

Since Hdeg $X \geq d+1$, there exists an $N+1$ point subset $A \subset X$ such that Hdeg $A \geq d+1$, by $\mathbf{1 . 2}$. This implies that $V(A) \neq 0$ (and $\operatorname{Hdeg} A=d+1$ ) by 2.5. Since $\varphi_{i_{1}}, \ldots, \varphi_{i_{k}}$ are similarity transformations, so is $\varphi_{i_{1}} \circ \cdots \circ \varphi_{i_{k}}$. Let $\lambda>0$ denote its similarity ratio. Of course, $\lambda<1$.

The set $A_{k}:=\left(\varphi_{i_{1}} \circ \cdots \circ \varphi_{i_{k}}\right)^{k}(A)$ is included in the closed ball of radius

$$
\lambda^{k} \cdot \max \{|\boldsymbol{x}-\boldsymbol{s}|: \boldsymbol{x} \in A\}
$$

with centre $\boldsymbol{s}$. If we number the points of $A_{k}$ as $A_{k}=\left\{\boldsymbol{a}_{0}^{k}, \ldots, \boldsymbol{a}_{N}^{k}\right\}$ arbitrarily, $A_{k}$ is contained in the ball of radius

$$
r_{k}:=2 \lambda^{k} \cdot \max \{|\boldsymbol{x}-\boldsymbol{s}|: \boldsymbol{x} \in A\}
$$

centred at $\boldsymbol{a}_{0}^{k}$. We know that

$$
\frac{\text { Det } V\left(A_{k}\right)}{\delta\left(A_{k}\right)^{n \cdot N(n+1, d-1)}}=\frac{\operatorname{Det} V\left(A_{1}\right)}{\delta\left(A_{1}\right)^{n \cdot N(n+1, d-1)}}
$$

by [5.1. Since this expression and the ratio $\delta\left(A_{k}\right) / r_{k}$ are independent of $k$, the condition (4) of the theorem holds.

Most of the classical fractal sets constructed geometrically are self-similar and not contained in an algebraic hypersurface (hence $\operatorname{Hdeg}(X) \geq d+1$ ). Among them are Cantor set, Koch curve, Sierpinski gasket and Menger sponge. Non-algebraicity of these fractal sets follows from the fact that the local Hausdorff dimension of a proper algebraic subset is smaller than that of the ambient space.

\section{Control of flatness by values}

Let $f$ be a $C^{d}$ function defined on an open neighbourhood of $0 \in \mathbb{R}^{n}$. Let us call $f k$-flat if $f^{(\boldsymbol{p})}(0)=0$ for $\boldsymbol{p}:=\left(p_{0}, \ldots, p_{n}\right) \in I(n, d)$ with $|\boldsymbol{p}| \leq k \leq d$.

Remark 6.1. As to the terms flatness and order, the author now understood that it is better to use both depending on the category of functions. When we treat analytic functions, order is convenient because it is a valuation (or related to valuations, on a singular space), a familiar notion to algebraists. If the order of $f$ is $p$, then $f$ is of course $(p-1)$-flat. When we treat $C^{d}$ functions for finite $d$, there occurs a difficulty in defining order. If all the partial derivatives of $f$ vanishes order up to $d, f$ is $d$-flat. But we can not define its order confidently, so long as we permit non-integer values. So flatness is better in this category. 
Theorem 6.2. Let $p>0$ be a positive number, $\left\{r_{k}\right\} \subset \mathbb{R}$ a positive sequence and $A_{k}:=\left\{\boldsymbol{a}_{0}^{k}, \ldots, \boldsymbol{a}_{N}^{k}\right\} \subset \mathbb{R}^{n}(k \in \mathbb{N})$ sets of $N(n, d)=N+1$ distinct points. Suppose that:

(1) $A_{k}$ is contained in the closed ball of radius $r_{k}$ centred at $\boldsymbol{a}_{0}^{k}$;

(2) $\lim _{k \rightarrow \infty} r_{k}=0$;

(3) $\lim _{k \rightarrow \infty} \boldsymbol{a}_{0}^{k}=0$;

(4) there exist $c, e>0$ such that $\left|\operatorname{Det} V\left(A_{k}\right)\right| \geq c \cdot r_{k}^{e}(k \in \mathbb{N})$.

For a $C^{d}(d \geq p)$ function $f$ defined in a neighbourhood of $\mathbf{0}$, we put

$S_{k}:=r_{k}^{-p} \cdot \max \left\{|f(\boldsymbol{x})|: \boldsymbol{x} \in A_{k}\right\}, \quad m:=p-(e-n \cdot N(n+1, d-1))$.

(i) If $m$ is an integer and $\lim _{k \rightarrow \infty} S_{k}=0$, then $f$ is $m$-flat at $\mathbf{0}$.

(ii) If $m$ is not an integer and $S_{k}$ is bounded, then $f$ is $[m]$-flat at $\mathbf{0}$, where $[\mathrm{m}]$ denotes the maximal integer not greater than $\mathrm{m}$.

Remark 6.3. This theorem is useful in the following situation. Let $\left\{s_{k}\right\} \subset \mathbb{R}$ be a positive sequence. Suppose that $A_{k}$ is contained in the closed ball of radius $s_{k}$ centred at $\mathbf{0}$. If $p, q>0, S_{k}$ in the theorem is majorized by

$$
T_{k}:=\frac{s_{k}^{q}}{r_{k}^{p}} \cdot \max \left\{\left|\frac{f(\boldsymbol{x})}{\boldsymbol{x}^{q}}\right|: \boldsymbol{x} \in A_{k}\right\} .
$$

The first factor of $T_{k}$ is concerned with the shrinking of balls containing $A_{k}$ and the second with flatness of the values of $f$ along $\bigcup A_{k}$. If the $T_{k}$ tend to 0 , then so do the $S_{k}$. If the $T_{k}$ are bounded, then so are the $S_{k}$.

Remark 6.4. The expression $m$ above is rather complicated. We can understand this as follows. If the conditions in the theorem holds, then $e$ must satisfy $e \geq$ $n \cdot N(n+1, d-1)$ by 2.2. The equality here means that the points of each $A_{k}$ are algebraically in general position in the balls of (1) "uniformly with respect to $k$ ". If this is the case, we have $m=p$ and, in view of 6.3. (i) is a sharpening of Spallek's theorem [S], 1.4: balls are replaced by sets $A_{k}$ of $N+1$ points. The term $e-n \cdot N(n+1, d-1) \geq 0$ is the adjustment for the case when the algebraic genericities of the positions of the points of $A_{k}$ degenerate as $k$ increases.

Proof of 6.2. We may assume that $f$ is defined in a neighbourhood of the closure of the convex hull of $\bigcup A_{k}$. Let us adopt an ordering of $I(n, d)$ such that

$$
\begin{gathered}
\{\boldsymbol{p}:|\boldsymbol{p}|<d\}=\left\{\boldsymbol{p}_{0}, \ldots, \boldsymbol{p}_{M}\right\}, \quad\{\boldsymbol{p}:|\boldsymbol{p}|=d\}=\left\{\boldsymbol{p}_{M+1}, \ldots, \boldsymbol{p}_{N}\right\} \\
(M=N(n, d-1)) .
\end{gathered}
$$


Then by the Taylor formula, there exists $\theta_{i}^{k} \in(0,1)$ such that we have

$$
V\left(A_{k}-\boldsymbol{a}_{\mathbf{0}}^{k}\right)\left(\begin{array}{c}
\frac{f^{\left(\boldsymbol{p}_{0}\right)}\left(\boldsymbol{a}_{0}^{k}\right)}{\boldsymbol{p}_{0} !} \\
\vdots \\
\frac{f^{\left(\boldsymbol{p}_{M}\right)}\left(\boldsymbol{a}_{0}^{k}\right)}{\boldsymbol{p}_{M} !} \\
\frac{f_{M+1}^{\left(\boldsymbol{p}^{\prime}\right)}\left(\boldsymbol{a}_{0}^{k}\right)}{\boldsymbol{p}_{M+1} !} \\
\vdots \\
\frac{f^{\left(\boldsymbol{p}_{N}\right)}\left(\boldsymbol{a}_{0}^{k}\right)}{\boldsymbol{p}_{N} !}
\end{array}\right)=\left(\begin{array}{c}
f\left(\boldsymbol{a}_{0}^{k}\right)+\delta_{1}^{k} \\
\vdots \\
f\left(\boldsymbol{a}_{M}^{k}\right)+\delta_{M}^{k} \\
f\left(\boldsymbol{a}_{M+1}^{k}\right)+\delta_{M+1}^{k} \\
\vdots \\
f\left(\boldsymbol{a}_{N}^{k}\right)+\delta_{N}^{k}
\end{array}\right),
$$

where

$$
\begin{gathered}
\delta_{i}^{k}:=\sum_{j=M+1}^{N}\left(f^{\left(\boldsymbol{p}_{j}\right)}\left(\boldsymbol{a}_{0}^{k}\right)-f^{\left(\boldsymbol{p}_{j}\right)}\left(\boldsymbol{b}_{i}^{k}\right)\right)\left(\boldsymbol{a}_{i}^{k}-\boldsymbol{a}_{0}^{k}\right)^{\boldsymbol{p}_{j}} / \boldsymbol{p}_{j} ! \\
\boldsymbol{b}_{i}^{k}:=\theta_{i}^{k} \boldsymbol{a}_{0}^{k}+\left(1-\theta_{i}^{k}\right) \boldsymbol{a}_{i}^{k}, 0<\theta_{i}^{k}<1 \quad(i=0, \ldots, N ; k=1,2, \ldots)
\end{gathered}
$$

and $V\left(A_{k}-\boldsymbol{a}_{0}^{k}\right)$ is the Vandermonde matrix of the translation of $A_{k}$ by $-\boldsymbol{a}_{0}^{k}$. As we have seen in the proof of 4.1 the elements with row index $i$ of

$$
\left(\operatorname{Det} V\left(A_{k}-\boldsymbol{a}_{0}^{k}\right)\right) V\left(A_{k}-\boldsymbol{a}_{0}^{k}\right)^{-1}
$$

are homogeneous of degree

$$
\sum_{j \neq i}\left|\boldsymbol{p}_{j}\right|=n \cdot N(n+1, d-1)-\left|\boldsymbol{p}_{i}\right|
$$

in all the components of all $\boldsymbol{a}_{i}-\boldsymbol{a}_{0}$. Hence there exists $C>0$ such that

$$
\left|f^{\left(\boldsymbol{p}_{i}\right)}\left(\boldsymbol{a}_{0}^{k}\right)\right| \leq C \cdot r_{k}^{m-p-\left|\boldsymbol{p}_{i}\right|} \max \left\{\left|f\left(\boldsymbol{a}_{i}^{k}\right)\right|+\left|\delta_{i}^{k}\right|: 1 \leq i \leq N\right\} .
$$

Sinse $f$ is of class $C^{d}$ and since $\lim _{k \rightarrow \infty} \boldsymbol{a}_{0}^{k}=\lim _{k \rightarrow \infty} \boldsymbol{b}_{i}^{k}=\mathbf{0}$, we see that

$$
\lim _{k \rightarrow \infty} r_{k}^{-d}\left|\delta_{i}^{k}\right|=0(1 \leq i \leq N) \text {. }
$$

Hence, if $\left|\boldsymbol{p}_{i}\right| \leq m$, we have

$$
\lim _{k \rightarrow \infty} r_{k}^{m-p-\left|p_{i}\right|}\left|\delta_{i}^{k}\right|=0 \quad(1 \leq i \leq N) .
$$

We have assumed that $\left|f\left(\boldsymbol{a}_{i}^{k}\right)\right| \leq r_{k}^{p} S_{k}$.

In the case of (i), this implies that $\lim _{k \rightarrow \infty}\left|f^{\left(\boldsymbol{p}_{i}\right)}\left(\boldsymbol{a}_{i}^{k}\right)\right|=0$ for $\boldsymbol{p}_{i}$ with $\left|\boldsymbol{p}_{i}\right| \leq$ $m$. Since $\lim _{k \rightarrow \infty} \boldsymbol{a}_{0}^{k}=\mathbf{0}$, we have $f^{\left(\boldsymbol{p}_{i}\right)}(\mathbf{0})=0$ for such $\boldsymbol{p}_{i}$, which completes the proof of (i).

In the case of (ii). let us define $\tilde{p}$ and $\tilde{S}_{k}$ by

$$
[m]=\tilde{p}-(e-n \cdot N(n+1, d-1)), \quad \tilde{S}_{k}:=r_{k}^{-\tilde{p}} \cdot \max \left\{|f(\boldsymbol{x})|: \boldsymbol{x} \in A_{k}\right\} .
$$


Since $\tilde{p}<p, \lim _{k \rightarrow \infty} \tilde{S}_{k}=0$ holds and (ii) follows from (i).

\section{REFERENCES}

[AS] A. A. Akopyan, A. A. Saakyan, Multivariate splines and polynomial interpolation, Russian Math. Surveys 48-5, 1-72 (1993).

$\left[\mathrm{BMP}_{1}\right]$ E. Bierstone, P. Milman, W. Pawłucki, Composite differentiable functions, Duke Math. J. 83, 607-620 (1996).

$\left[\mathrm{BMP}_{2}\right]$ E. Bierstone, P. Milman, W. Pawłucki, Differentiable functions defined in closed sets, A problem of Whitney, Inventiones Math. 151, 329-352 (2003).

[B] G. Bouligand, Introduction à la géométrie infinitésimale directe, Vuibert, Paris (1932).

[C] G. Choquet, Convergence, Ann. Inst. Fourier, 23 57-112 (1948).

[F] K. Falconer, Techniques in fractal geometry, John-Wiley and Sons, 1997.

$\left[\mathrm{G}_{1}\right] \quad$ G. Glaeser, Études de quelques algèbres tayloriennes, J. Analyse Math. 6 1-124 (1958).

$\left[\mathrm{G}_{2}\right]$ G. Glaeser, L'interpolation des fonctions différentiables de plusieurs variables, in: Proceedings of Liverpool singularities symposium II (ed. C. T. C. Wall) (LMN 209), Springer, Berlin, 1971, 1-33.

[I] S. Izumi, Flatness of differentiable functions along a subset of a real analytic set, J. Analyse Math. 86, 235-246 (2002).

[K] P. Kergin, A natural interpolation of $C^{K}$ functions, J. Approximation Theory 29, 278$293(1980)$.

$[\mathrm{MM}] \quad$ C. A. Micchelli, P. Milman, A formula for Kergin interpolation in $\mathbb{R}^{k}$, J. Approximation theory 29, 294-296 (1980).

[S] K. Spallek, l-Platte Funktionen auf semianalytischen Mengen, Math. Ann. 227, 277-286 (1977).

$\left[\mathrm{W}_{1}\right] \quad \mathrm{H}$. Whitney, Analytic extensions of differentiable functions defined in closed sets, Trans. Amer. Math. Soc. 36, 63-89 (1934).

$\left[\mathrm{W}_{2}\right] \quad$ H. Whitney, Differentiable functions defined in closed sets. I, Trans. Amer. Math. Soc. 36-2, 369-387 (1934).

[YHK] M. Yamaguti, M. Hata, J. Kigami, Mathematics of Fractals (Translations Math. Monographs 167), AMS, Providence 1997.

Department of Mathematics

Kinki University

Kowakae Higashi-Osaka 577-8502, Japan

e-mail: izumi@math.kindai.ac.jp 